Musées, Patrimoine et Culture scientifiques et techniques

\title{
Le Jardin des sciences de Dijon au cœur des enjeux liés à la biodiversité
}

\section{Agnès Fougeron et Gérard Ferrière}

\section{(2) OpenEdition \\ Journals}

Édition électronique

URL : http://journals.openedition.org/ocim/1510

DOI : 10.4000/ocim. 1510

ISSN : 2108-646X

\section{Éditeur}

OCIM

\section{Édition imprimée}

Date de publication : 1 mai 2015

Pagination : 12-15

ISSN : 0994-1908

\section{Référence électronique}

Agnès Fougeron et Gérard Ferrière, «Le Jardin des sciences de Dijon au cœur des enjeux liés à la biodiversité », La Lettre de l'OCIM [En ligne], 159 | 2015, mis en ligne le 01 mai 2016, consulté le 01 mai 2019. URL : http://journals.openedition.org/ocim/1510 ; DOI : 10.4000/ocim.1510

Ce document a été généré automatiquement le 1 mai 2019.

Tous droits réservés 


\title{
Le Jardin des sciences de Dijon au cœur des enjeux liés à la biodiversité
}

\author{
Agnès Fougeron et Gérard Ferrière
}

\section{LE JARDIN BOTANIQUE DE DIJON}

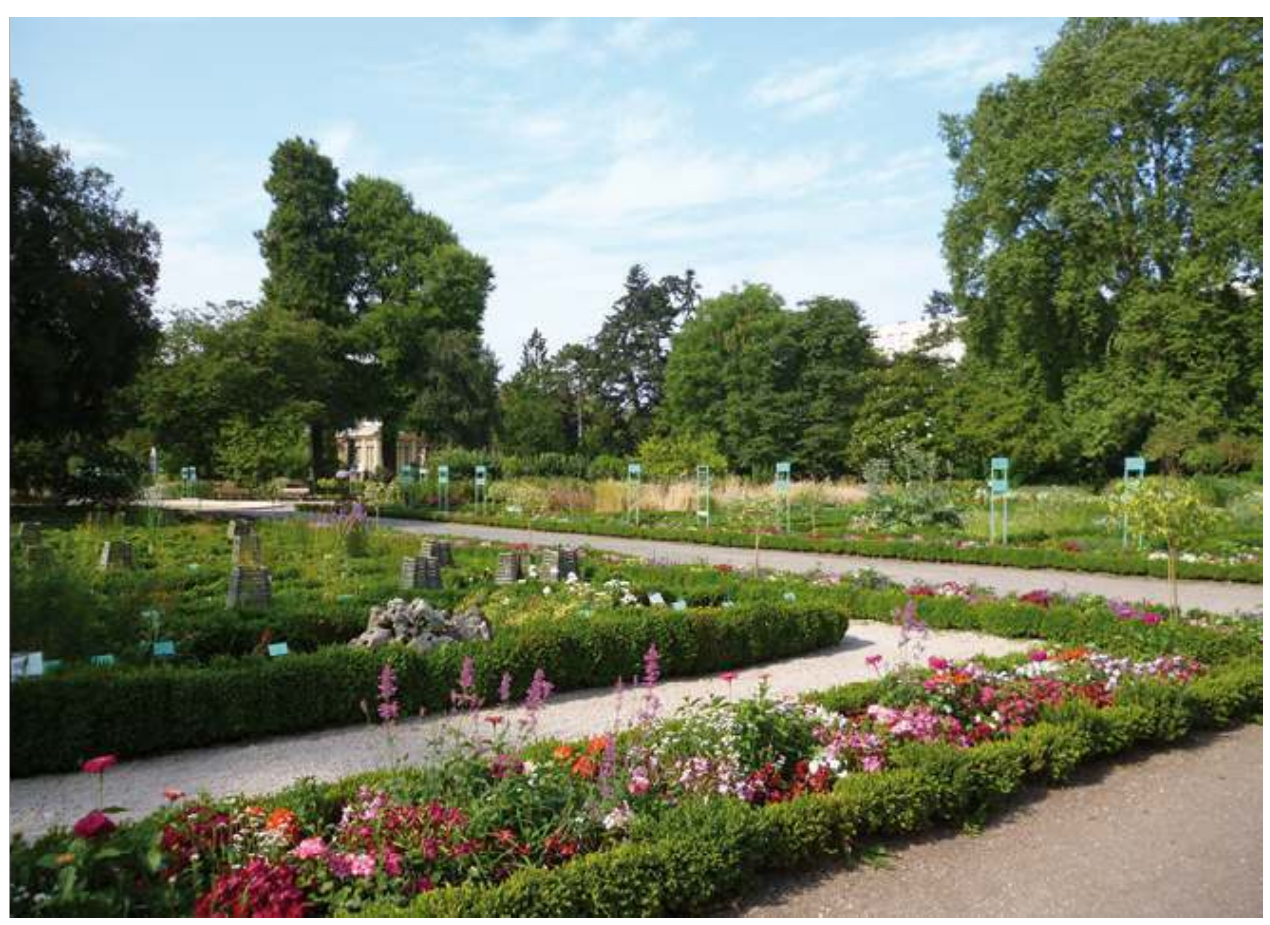

(c) Jardin des sciences

1 Le Muséum-Jardin des sciences de la ville de Dijon est un lieu de diffusion des savoirs scientifiques consacrés aux sciences de la nature et aux relations entre l'homme et 
l'environnement. Structure originale recevant près de 200000 visiteurs par an, elle regroupe un muséum d'Histoire naturelle $\left(1000 \mathrm{~m}^{2}\right.$ d'expositions, 2000 spécimens présentés au public au sein de l'exposition permanente), un jardin botanique (une superficie de 5 hectares comprenant 1500 espèces et variétés florales et 250 essences d'arbres différentes) et un planétarium (d'une capacité de 60 places assurant plus de 500 heures de projection par an).

2 Le projet d'établissement réaffirme l'identité et le positionnement de l'institution et fait le pari de revenir à ses fondamentaux d'origine : connaissance et partage du vivant dans toute sa diversité, accompagnés d'un ancrage régional fort.

3 La prise en compte de la «nature urbaine » est aujourd'hui un élément structurant de l'action municipale. C'est une donnée récente et ses usages directs ou indirects suscitent une approche pluridisciplinaire. Un plan biodiversité a été mis en place, il est animé par le Muséum-Jardin des sciences en transversalité avec les services de la ville de Dijon et de la communauté urbaine. Le pilotage politique est assuré par un collège d'élus.

4 Ce plan développe et fédère les connaissances sur la biodiversité urbaine, porte les messages auprès de tous les acteurs. Il propose de développer une «culture de la biodiversité » au sein de la collectivité à partir d'axes thématiques qui s'intègrent aux problématiques régionales et nationales: Schéma Régional de Cohérence Écologique, Trames vertes et bleues et sa déclinaison locale, Système d'Information sur la Nature et les Paysages en Bourgogne, mise en place de la Stratégie régionale pour la biodiversité et la mise en œuvre d'un Observatoire régional, signature de la charte « Zéro phyto » et ses implications dans les concepts d'aménagement et de gestion du végétal en ville, implication des citoyens et développement des sciences participatives...

\section{Le Jardin des sciences participe à la Nuit des Chercheurs.}

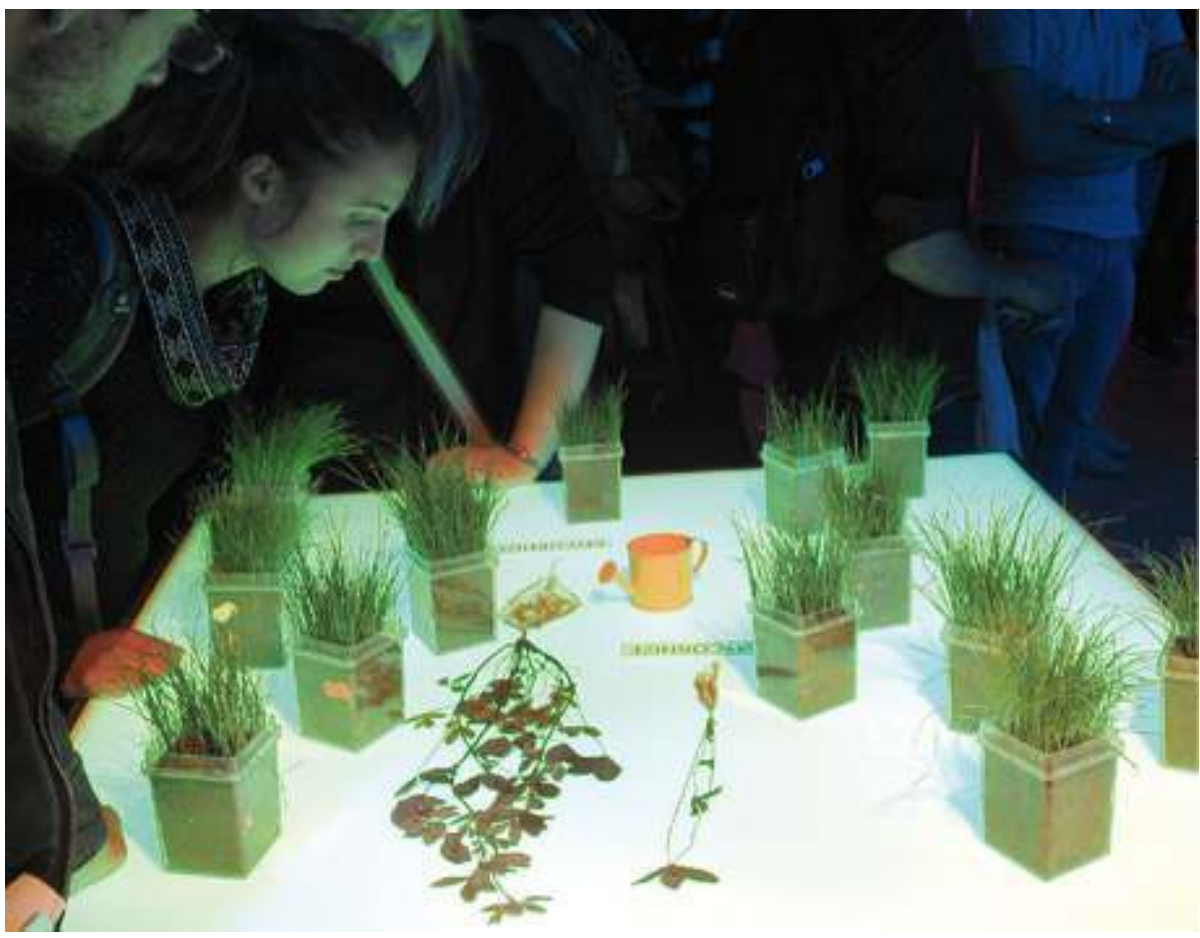

(c) Agroécologie 
Une expérimentation sur la végétalisation des lignes de tramway menée en collaboration avec l'unité mixte de recherche Agroécologie.

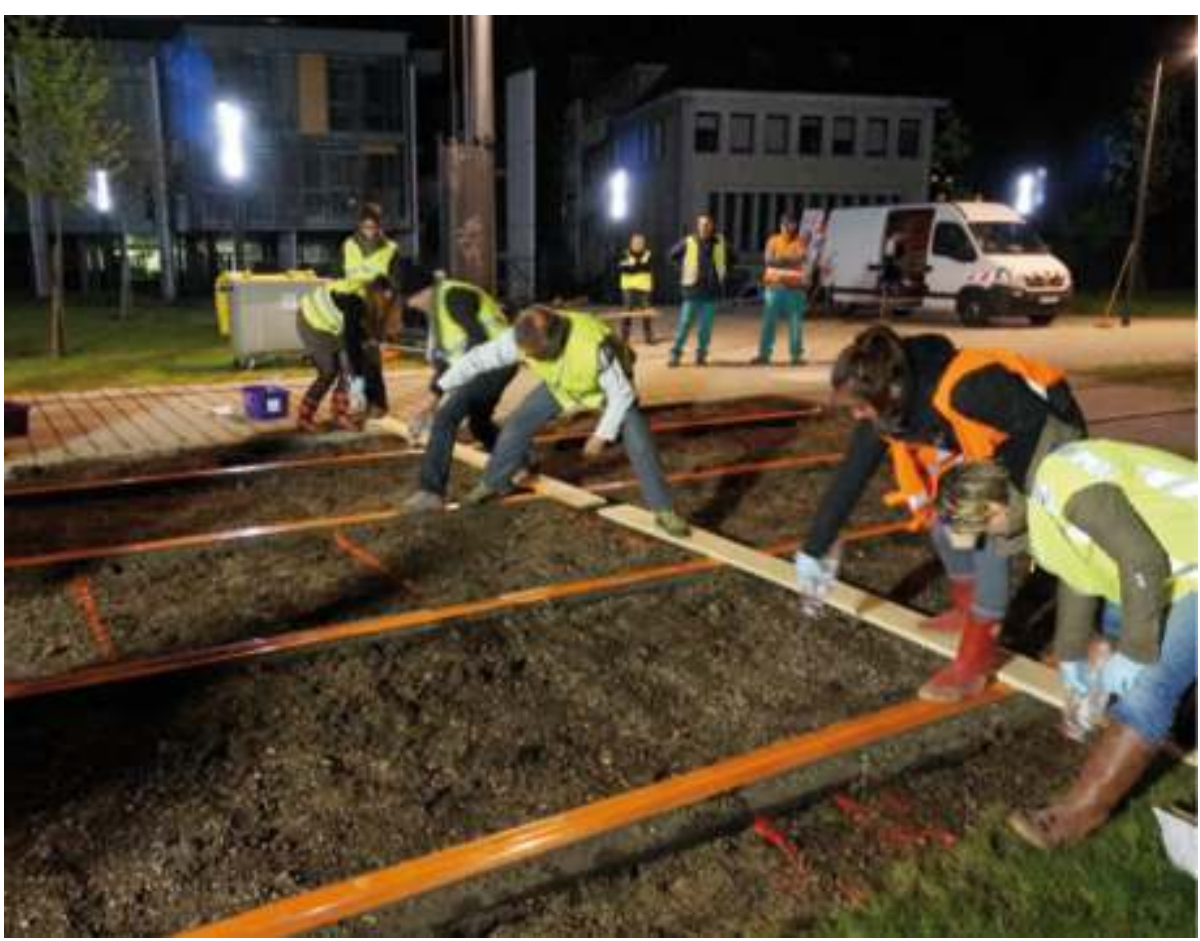

(c) Jardin des sciences

Participation à l'opération « Sauvages dans ma rue » qui recense la flore urbaine.

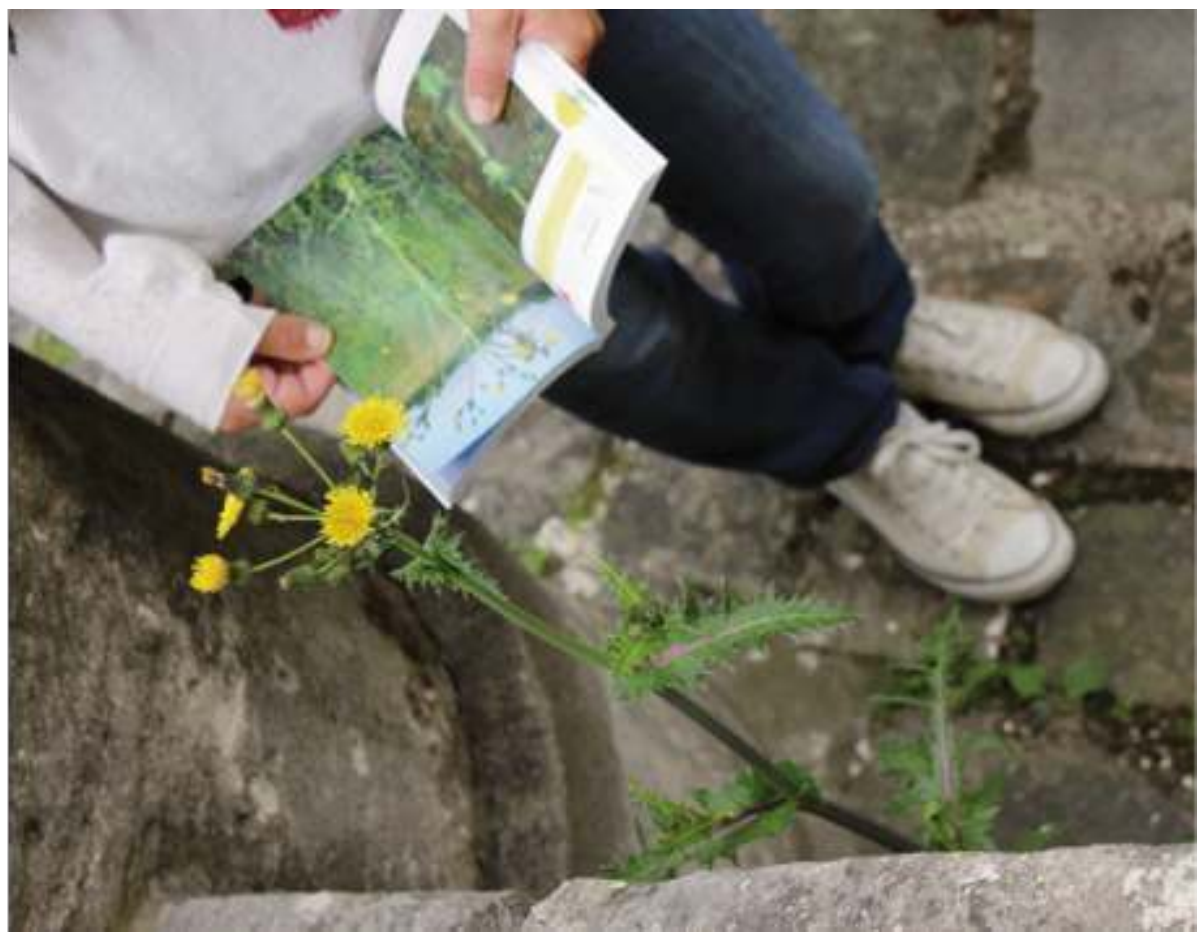

(c) MNHN/ M. Evanno

5 Concrètement, le Jardin des sciences coordonne les actions suivantes : 
6 - l'identification et la hiérarchisation des réservoirs écologiques, repérage des corridors potentiels et de leur intégration éventuelle dans le cadre du réseau urbain concernant les déplacements doux ;

7 - la prise en compte de la nature en ville dans le cadre des aménagements urbains (exemple écoquartiers, façades et toitures végétalisées, prairies urbaines écologiques, prairies fleuries...);

8 - la mise en place d'expérimentations avec les pôles de recherches locaux : en partenariat avec l'unité mixte de recherche Agroécologie (INRA/Agrosup/université de Bourgogne), étude de nouvelles modalités de végétalisation des linéaires de tramway en alternative aux gazons de graminées conventionnels. L'expérimentation consiste en la mycorhization de plants sélectionnés (micro-trèfle, micro-luzerne...). L'objectif est de minimiser l'impact environnemental et de favoriser la biodiversité urbaine (en lien avec les corridors écologiques de la Trame verte urbaine), notamment par une végétalisation spécifique des abords des plateformes de roulement ;

9 - l'expertise apportée sur des projets structurants de la collectivité (élaboration de la Cité internationale de la Gastronomie, agriculture périurbaine, programme «miel de Dijon » et mise en place de ruches) ;

10 - le développement des actions citoyennes, au niveau de la connaissance et de l'appropriation des enjeux :

11 - «J'adopte un arbre, un pied d'immeuble » : par l'intermédiaire des commissions de quartiers mises en place par le service démocratie locale, les habitants sont invités à choisir des ensembles d'arbres ou de micro-zones à végétaliser, à en assurer l'entretien. Le Jardin des sciences avec le service des espaces verts, anime les groupes de travail, guide la sélection des essences implantées dans les zones à enjeux écologiques, fournit les végétaux...;

12 - jardins partagés : outre leur fonction traditionnelle de développement de lien social au sein des quartiers, l'objectif est de développer la biodiversité sauvage et cultivée (conservation de cultivars menacés par parrainage et échanges de graines, aménagement dans les parties communes de zones favorisant par exemple les pollinisateurs et les auxiliaires des cultures) ;

13 - jardins pédagogiques: de nombreux «jardins au carrés » sont disséminés au sein des groupes scolaires de la ville. Les médiateurs du Jardin des sciences intègrent ces outils dans les activités éducatives proposées et servent de relais avec les services techniques de la ville, traditionnels gestionnaires de ces équipements ;

14 - programmes de sciences participatives : le Jardin des sciences s'associe aux démarches de science participative menées par le Muséum national d'Histoire naturelle (programmes Vigie-Nature). À l'échelle de la collectivité, il relaie les programmes Sauvages de ma rue consacré à la flore spontanée urbaine, Opération escargot et SPIPOLL ou suivi photographique des insectes pollinisateurs. Le Jardin des sciences accompagne les observateurs volontaires et restitue les résultats, à partir notamment d'approches culturelles variées ;

15 - mise en place d'un portail Internet dédié à la biodiversité urbaine, en lien avec les sciences participatives. À partir de juin 2015, le site www.ma-nature.dijon.fr présente les initiatives locales, anime le réseau des participants aux programmes Vigie-Nature, fait connaître les activités du Jardin des sciences et de la collectivité sur cette thématique. 
Installation de ruches au cœur du Jardin botanique.

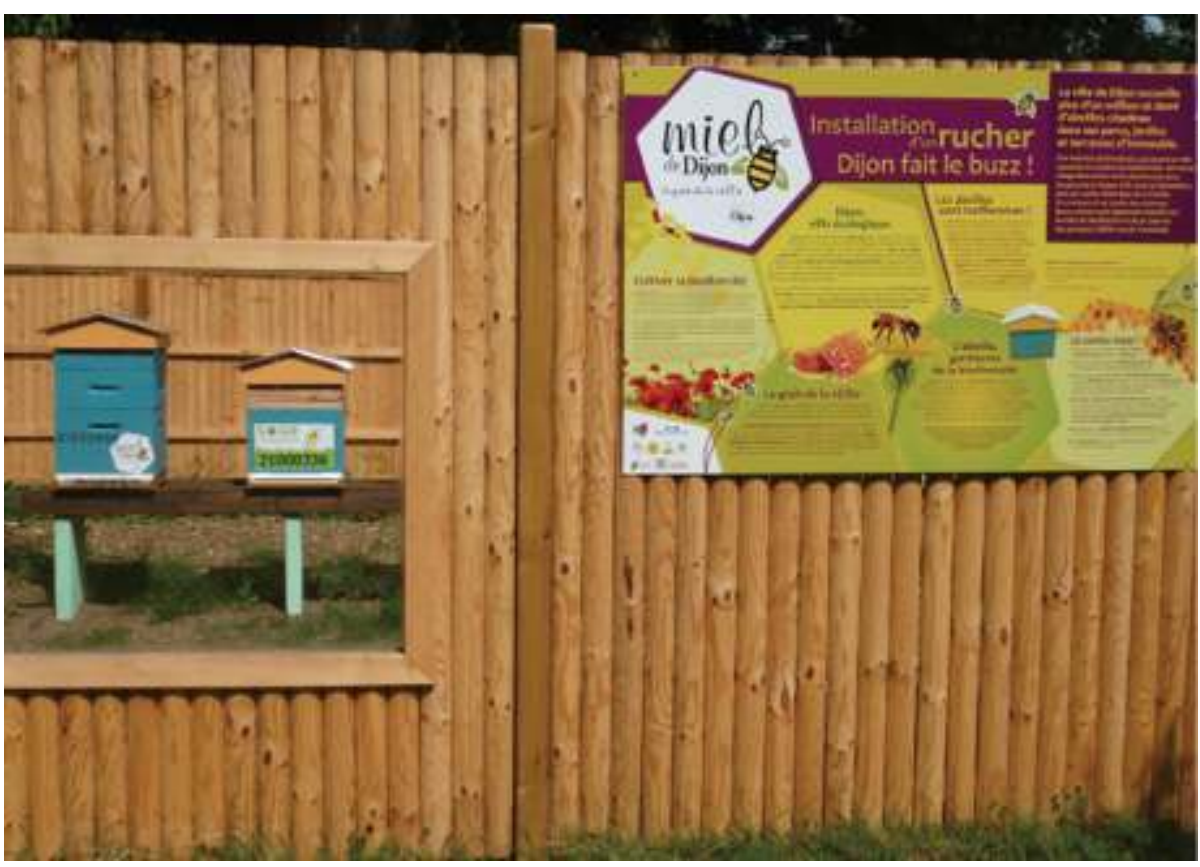

(c) Jardin des sciences

Le Plan Biodiversité se traduit pour le Jardin des sciences par des opérations ex situ, sur le territoire de la collectivité, mais également sur le site même de l'institution (le Jardin de l'Arquebuse), où une rénovation complète de l'établissement s'est déroulée. Ainsi, les équipements proposés offrent aujourd'hui une vision complète et transverse du vivant :

- Le Planétarium numérique propose spectacles en immersion d'images et ateliers pour comprendre l'Univers, le système solaire, la formation de la Terre où la vie a pu se développer ; il est complété par une exposition permanente ;

18 - Le Muséum, entièrement rénové, aborde l'apparition de la vie, sa diversification, et son expression dans la biodiversité actuelle, notamment dans la région Bourgogne. Le parcours muséographique se termine en abordant la place de l'humain et ses impacts sur la biodiversité ;

19 - Le Jardin botanique est aujourd'hui totalement dédié à la diversité végétale, sauvage et cultivée. Il offre un espace de plein air complémentaire aux thématiques abordées dans l'exposition permanente du Muséum.

20 Les expositions temporaires et les activités événementielles programmées contribuent à cette dynamique générale. L'établissement s'investit aujourd'hui fortement sur deux opérations nationales : la « Fête de la nature » et les journées nationales «API days » dans le cadre du programme « Abeille, sentinelle de l'environnement ». 


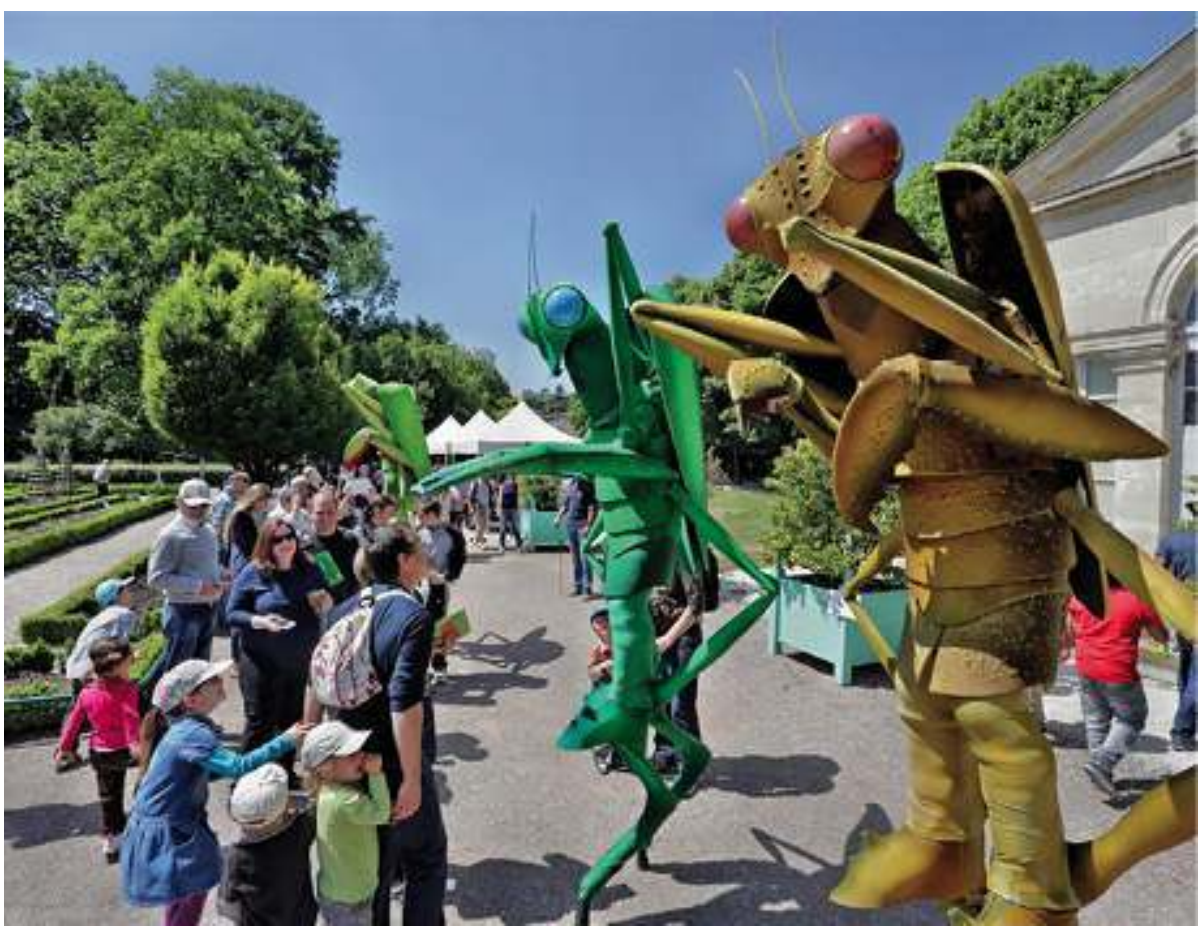

(c) Ville de Dijon

21 Le projet d'établissement a également porté sur la priorisation des projets conduits dans les différents domaines d'activités de l'institution, notamment au niveau du patrimoine naturel en collections. L'objectif "biodiversité » est réaffirmé dans le sens et le rôle donnés aux collections: témoins d'un état de la biodiversité passée, des recherches conduites sur le terrain complètent ces données naturalistes. Les collections participent à la compréhension des dynamiques des écosystèmes, notamment urbains (exemple : place du végétal en ville, développement de la flore spontanée: programmes conduits en partenariat avec l'antenne du Conservatoire Botanique National).

Aujourd'hui, ces investigations concernent principalement la zone géographique de la collectivité (Grand Dijon), des zones à enjeux identifiés dans le cadre du SRCE (Schéma régional de cohérence écologique) et de la TVB (Trames vertes et bleues) régionale et des réserves naturelles situées à proximité. Les informations de terrain sont récoltées directement par les scientifiques du Jardin des sciences ainsi qu'à partir des programmes de sciences participatives, associant ainsi les citoyens aux enjeux. Les données naturalistes, issues des collections ou des observations actuelles sont intégrées via notamment la plate-forme numérique e-ReColNat à la base régionale «système d'information sur la nature et les paysages de Bourgogne » de la DRÉAL, qui alimente les bases de données naturalistes nationales et internationales.

En parallèle sur le site de l'établissement, des modes de gestion plus respectueux de l'environnement sont mis en place (compostage, tri sélectif des déchets pour les publics, régulation climatique du muséum par géothermie, connexion du planétarium au réseau de chaleur urbain, économies d'eau...).

Le Plan Biodiversité 


\section{RÉSUMÉS}

Les muséums d'Histoire naturelle et les jardins botaniques reflètent les découvertes naturalistes et l'évolution des concepts scientifiques, ils témoignent des changements et des attentes globales de la société : le Muséum-Jardin des sciences de Dijon prend en compte cette dimension notamment pour ce qui concerne la protection de la «nature urbaine » en coordonnant le plan biodiversité élaboré par la Ville de Dijon et la communauté urbaine.

\section{INDEX}

Mots-clés : biodiversité, jardin botanique, muséum 


\section{AUTEURS}

\section{AGNÈS FOUGERON}

directrice adjointe au Jardin des sciences de Dijon afougeron@ville-dijon.fr

\section{GÉRARD FERRIÈRE}

directeur du Jardin des sciences de Dijon gferriere@ville-dijon.fr 